\title{
Stiffness Modulation of A1-DOF Robotic Manipulator with Antagonistic Actuators
}

\author{
J.H. Lee \\ Department of Mechanical Engineering \\ Graduate School of Science and Engineering \\ Ehime University \\ Japan
}

\author{
S. Okamoto \\ Department of Mechanical Engineering \\ Graduate School of Science and Engineering \\ Ehime University \\ Japan
}

\begin{abstract}
Stiffness modulation scheme for a robotic driving system with redundant actuation was investigated in this paper. The dynamic and the stiffness models of a simple system having one link and two prismatic actuators were derived and employed for computational simulations of dynamic response to an initial disturbance. Several combinations with different types of elastic elements were applied to the system and their movement were analysed.
\end{abstract}

Keywords-stiffness modulation; redundant actuation; antagonistic stiffness; musculoskeletal structure

\section{INTRODUCTION}

One of the different aspects of biological systems like as human body from general robotic systems is the fact that their behaviours are so smart and soft to adapt themselves to various environmental situations. It has been known that it is based on their inherent characteristic due to compliant actuator, i.e. muscle, which can adjust its impedance different from the stiff actuation mechanism using typical motor with gear set of high reduction ratio [1]. In order to cope with the limitation of general robots, researches to endow robots with intelligent soft property have been carried out recently. Researches to develop a special joint having the function to change its stiffness have been performed, and useful devices and its applications also have been reported [2, 3]. On the other hand, research works to develop a biomimetic robot having soft characteristics based on musculoskeletal structure and antagonistic multiple actuators have been done [4]. It has been explained that the musculoskeletal structure with antagonistic actuation provides the capability of stable and fast motion in spite of the limited bandwidth of neuro feedback and sensor characteristics [1]. Besides, the nonlinear stiffness of actuators in the bio-system was known as an essential property for changing the system impedance. Therefore, the goal of this research is to investigate the effect of nonlinearity in musculoskeletal system from a view point of stiffness. For that, the simple driving unit of musculoskeletal structure was modelled and its dynamic response was analysed through computational simulation works.

\section{SYSTEM MODEL}

\section{A. Dynamic Model}

Figure I shows a 1-DOF robotic manipulator based on the musculoskeletal structure. Its motion is generated by the co- activation of both prismatic actuators, i.e. muscles in biological systems. It is a simple system having only one link and two mono-articular actuators, but a basic unit that can be employed to the general multi-DOF manipulator with biarticular actuators like as biological systems [5]. Wherem and I denote the mass and the inertia of the link, $\mathrm{d}_{1}$ andd $\mathrm{d}_{\mathrm{r}}$ denote the lengths of bothprismatic actuators, $l_{1}$ and $l_{2}$ denote the positions of insertions, respectively. The dynamic model including the assumed damping effect $c_{a}$ at the joint $\theta_{a}$ is given as follow

$$
\left(\mathrm{I}+\mathrm{ml}_{2}^{2}\right) \ddot{\theta}+\mathrm{c}_{\mathrm{a}} \dot{\theta}=\tau_{\mathrm{a}}^{*}
$$

Where it was assumed that the system is placed horizontally, so the gravity effect is not considered in this study. Besides, the mass and the inertia of prismatic actuators were neglected.

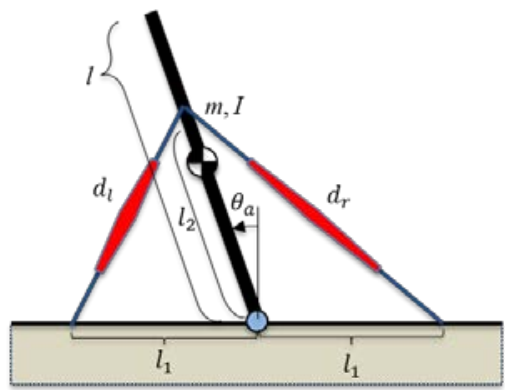

FIGURE I. ROBOTIC MANIPULATOR WITH TWO ANTAGONISTIC ACTUATORS.

\section{B. Stiffness Model}

When the system is in the state of static equilibrium, the effective torque of the independent joint $\theta_{a}$ is

$$
\tau_{\mathrm{a}}^{*}=\tau_{\mathrm{a}}+\left[\mathrm{G}_{\mathrm{a}}^{\mathrm{p}}\right]^{\mathrm{T}} \mathrm{T}_{\mathrm{p}}=0 .
$$

Where $\tau_{a}$ denotes the actuator torque applied to the independent joint, $T_{p}$ denotes the force (or torque in rotational joint case) of dependent joints, $\left[G_{a}^{p}\right]$ is the Jacobian which relates the dependent joints $\theta_{p}$ to the independent input joint $\theta_{a}$, respectively. When an external disturbance is applied to the system, an effective restoring torque is generated and 
causes spring-like behaviour resultantly. It can be modeled with respect to the independent joint of the system as [6]

$$
\Delta \tau_{\mathrm{a}}^{*}=-\left[\mathrm{K}_{\mathrm{aa}}\right] \Delta \theta_{\mathrm{a}}
$$

The system stiffness $\left[K_{a a}\right]$ is defined as

$$
\begin{gathered}
{\left[\mathrm{K}_{\mathrm{aa}}\right]=-\frac{\partial \tau_{\mathrm{a}}^{*}}{\partial \theta_{\mathrm{a}}}=\left[\mathrm{K}_{\mathrm{aa}}^{\mathrm{F}}\right]+\left[\mathrm{G}_{\mathrm{a}}^{\mathrm{p}}\right]^{\mathrm{T}}\left[\mathrm{K}_{\mathrm{pp}}^{\mathrm{F}}\right]\left[\mathrm{G}_{\mathrm{a}}^{\mathrm{p}}\right]+} \\
\left(-\mathrm{T}_{\mathrm{p}}\right)^{\mathrm{T}} \mathrm{o}\left[\mathrm{H}_{\mathrm{aa}}^{\mathrm{p}}\right] .
\end{gathered}
$$

Where the first and the second terms denote serial combinations of the passive joint stiffness and the feedback stiffness at the independent and dependent joints, respectively. The third term is the antagonistic stiffness generated by redundant actuation. It can be utilized for the modulation of system stiffness in parallel mechanism having closed chains with redundant actuators. Here, $\left[H_{a a}^{p}\right]$ denotes the Hessian relating the dependent joints $\theta_{p}$ to the independent input joint $\theta_{a}$.

\section{STIFFnESS MODUlATION SCHEMES}

Based on Eq. (4), the stiffness of the system can be generated by using the following methods.

Firstly, attaching a torsional spring between the link and the motor axis at the independent joint could be an easy way to provide stiffness to the system. However, for changing the stiffness according to various situations, a special spring component having the function to adjust joint stiffness is needed [2].

Secondly, instead of a torsional spring, a feedback control using the motor at the independent joint and the angle measurement could be employed like as general robots. However, general motors providing high torque for the expected motion have no back-drive ability due to high reduction ratio and friction property in its gear set. So a special motor of direct drive actuation or low reduction ratio is required. Besides, an intelligent control algorithm is also needed for changing stiffness according to the situations. Consequently, because of above mentioned limitations of the scheme using spring and typical feedback control, a special joint actuator having both functions of motion control and stiffness adjustment has been developed recently [2].

Thirdly, typical tension springs could be employed in connecting both prismatic joints. In this case, the restoring force proportional to the angular error from the equilibrium position is generated like as the case of the system with a torsional spring at the independent joint. Based on the appearance, it seems like that this scheme is similar to biological systems with antagonistic redundant actuation of muscles, so it has the capability to adjust system stiffness by changing pulling force of prismatic actuators. However, the resultant system stiffness cannot be changed in large amount even though the pulling preload forces of both actuators are changed because it is dependent to the unchanging constant of tension springs [1]. Therefore, for changing the system stiffness, a special tension spring with the function to adjust stiffness of itself is also required for this design.
Fourthly, the combination of a tension spring with passive nonlinear stiffness and a prismatic actuator can be another effective feed forward scheme for modulating system stiffness. If a special nonlinear tension spring whose stiffness increases according to its extended length is employed as the tendon connecting actuators, the system stiffness can be increased by applying preload forces of prismatic actuators in feed forward manner. It is coincident with the usual behaviour of biological system whose stiffness is strengthened with the increase of internal force of redundant muscles. In addition, this scheme can be applied as an effective control method incorporating feedback control with position measurement.

Finally, the antagonistic stiffness defined as the third term of Eq. (4) is a method to modulate the system stiffness. Even in the case there is no passive elastic component like as spring in the system, its stiffness can be modulated by the redundant actuation forces in closed loop structure [5]. Namely, the system stiffness could be strengthen by raising the actuation forces, which is also similar to the behaviour of biological systems.

\section{COMPUTATIONAL SimUlATION}

The characteristics of the systems with the above mentioned stiffness modulation schemes were investigated through computer simulations in this section. The motion responses to an initial disturbance were simulated by using the model described in the previous section. The equilibrium position was set to $0[\mathrm{rad}]$ and the initial disturbance of $0.2[\mathrm{rad}]$ was given to the system. The parameters used in the computation model displayed in Figure I are given as follows: $0.1[m]$.

$m=1[\mathrm{~kg}], I=0.0142\left[\mathrm{kgm}^{2}\right], l=0.5[\mathrm{~m}]$, and $l_{1}=l_{2}=$

Besides, the effective damping coefficient at the independent joint was assumed as $0.1[\mathrm{Nm} \mathrm{s} / \mathrm{rad}]$.

Figure II shows the behaviour of the system with passive springs when the position error is given as the initial disturbance. Both resultant motions of the systems with one torsional spring at the independent joint (dashed line) and two linear springs in the prismatic joints (solid line) show almost same motion trajectories. Where the spring coefficient of the torsional spring was set to $1[\mathrm{Nm} / \mathrm{rad}]$. The linear springs with the stiffness of $230[\mathrm{~N} / \mathrm{m}]$ was employed for the same effect of the torsional spring at the independent joint in this research. Its resultant force related to displacement is shown with solid line in FigureIII. 


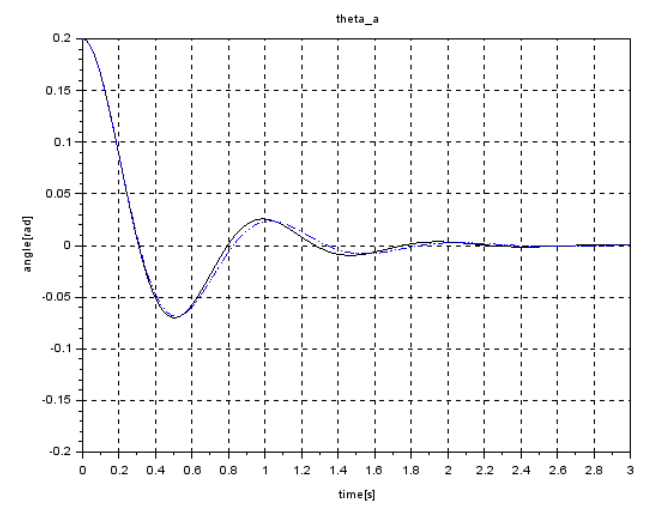

FIGURE II. MOTION TRAJECTORIES OF THE SYSTEM WITH A TORSIONAL SPRING AT THE JOINT $\Theta_{A}$ (DASHED LINE) AND THAT WITH TENSION SPRINGS AT BOTH PRISMATIC JOINTS (SOLID LINE)

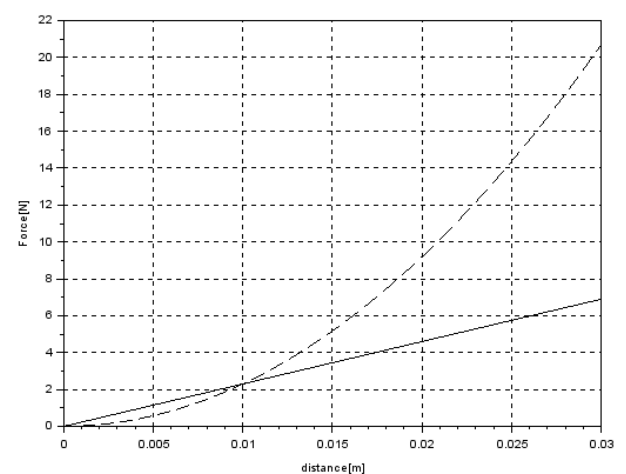

FIGURE III. THE RELATIONSHIP BETWEEN DISPLACEMENT AND RESTORING FORCE OF THE LINEAR SPRING (SOLID LINE) AND THE NONLINEAR SPRING (DASHED LINE) USED IN THE SIMULATIONS

Besides, the effect of the stiffness change of the system with linear springs under preload was investigated. Here, the preloads of 2.3N (dashed line) and 6.9[N] (solid line) were applied both linear springs, and the resultant trajectories are given in Figure IV. It is observed that the preloads of both linear springs enlarge the stiffness property of the system.

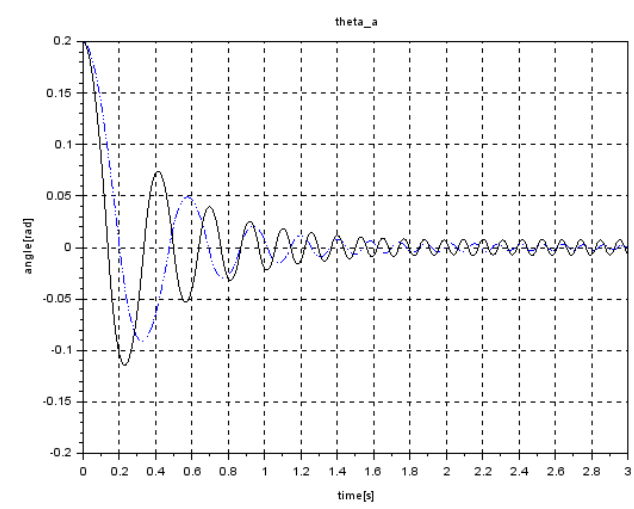

FIGURE IV. MOTION TRAJECTORIES OF THE SYSTEM WITH LINEAR SPRINGS UNDER PRELOAD OF 2.3[N] (DASHED LINE) AND 6.9[N] (SOLID LINE)

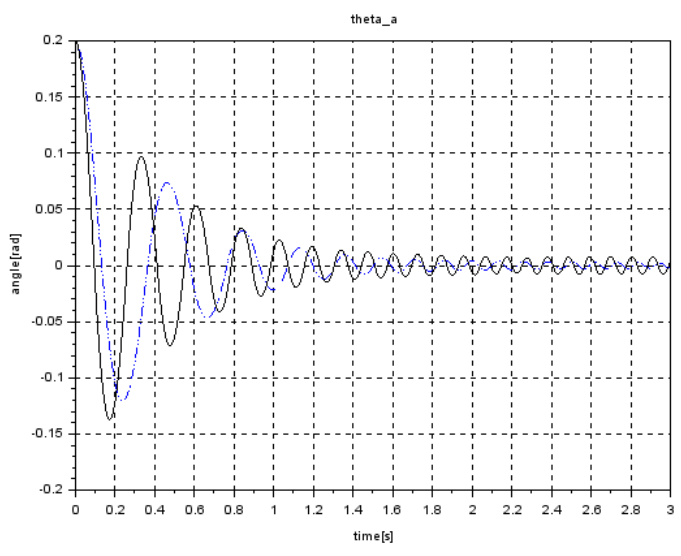

FIGURE V. MOTION TRAJECTORIES OF THE SYSTEM WITH NONLINEAR SPRINGS UNDER PRELOAD OF 2.3[N] AND 6.9[N]

Finally, in order to investigate the effect of the nonlinear property of the spring for the stiffness modulation, the motion of the system having nonlinear springs in both prismatic joints was simulated for the same situation with the previous simulation. The property of nonlinear spring used in this research is given as the dashed line in FigureIII. The resultant motion is given in Figure V. Similar to the previous case of linear spring, it has been observed that the higher preload provides the bigger stiffness to the system and its response becomes faster resultantly. Besides, the effect of nonlinear spring to raise the stiffness of the system is more successful than that of linear spring.

\section{CONCLUSION}

Several schemes of the stiffness modulation for 1-DOF robotic manipulator were investigated through computational simulations based on the stiffness model including redundant actuation. It was concluded that the biomimetic structure including multiple bi-articular actuators connected to elastic tendon with nonlinear property is adequate for effective modulation of the system stiffness than others. This result gives meaningful information that, in cooperation with muscle actuators of excellent capability, the musculoskeletal structure and the tendon of nonlinear property play an important role in stiffness modulation.

\section{REFERENCES}

[1] Hogan, N., Adaptive control of mechanical impedance by coactivation of antagonist muscles, IEEE Transactions on Automatic Control, (29)8, 1984.

[2] De Luca, A., Flacco F., Bicchi, A., Schiavi, R., Nonlinear decoupled motion-stiffness control and collision detection/reaction for the VSA-II variable stiffness device, Proc. of 2009 IEEE/RSJ International Conference on Intelligent Robots and Systems, pp. 5487-5494, 2009.

[3] English, C. E., and Russel, D., Mechanics and stiffness limitations of a variable stiffness actuator for use in a prosthetic limbs, (34), pp. 7-25, 1999.

[4] Jafari, A., Tsagarakis, N. G.,Sardellitti, I., and Caldwell, D. G., How design can affect the energy required to regulate the stiffness in variable stiffness actuators, Proc. of 2012 IEEE International Conference on Robotics and Automation, pp. 2792-2797, 2012. 
[5] Lee, J. H., and Yi, B.-J., Modulation of dynamic behavior of anthropomorphic robot: A biomimetic approach with force redundancy, Mechatronics, (21), pp. 261-271, 2011.

[6] Yi, B.-J., and Freeman, R. A.,Geometric analysis of antagonistic stiffness in redundantly actuated parallel mechanisms; special issues on parallel closed-chain mechanism. Journal of Robotic Systems, (10)5,pp. 581-603, 1993. 